\title{
Erratum: Elements of a theory for multiparton interactions in QCD
}

Markus Diehl, ${ }^{a}$ Daniel Ostermeier ${ }^{b}$ and Andreas Schäfer ${ }^{b}$

${ }^{a}$ Deutsches Elektronen-Synchroton DESY, 22603 Hamburg, Germany

${ }^{b}$ Institut für Theoretische Physik, Universität Regensburg, 93040 Regensburg, Germany

E-mail: markus.diehl@desy.de,

Daniel.Ostermeier@physik.uni-regensburg.de,

Andreas.Schaefer@physik.uni-regensburg.de

ERratum TO: JHEP03(2012)089

ARXIV EPRINT: 1111.0910

1. In equations (3.41) and (3.42), all terms $v^{+} v^{-}$should be changed into $v^{+} w^{-}$.

2. The second part of equation (4.25) correctly reads

$$
M_{a_{1}, a_{2}}^{n_{1}, n_{2}}\left(\boldsymbol{y}^{2}\right)=\frac{1}{2}\left(p^{+}\right)^{1-n_{1}-n_{2}} \int d y^{-}\left\langle p\left|\mathcal{O}_{a_{1}}^{+\cdots+}(0) \mathcal{O}_{a_{2}}^{+\cdots+}(y)\right| p\right\rangle_{y^{+}=0}
$$

with a factor $1 / 2$ instead of 2 .

3. Equation (5.75) correctly reads

$$
\begin{aligned}
{\left.\left[\prod_{i=1}^{2} \int d^{2} \boldsymbol{k}_{i}\right]{ }^{1} F_{a_{1}, \bar{a}_{2}}\left(x_{i}, \boldsymbol{k}_{i}, \boldsymbol{r}\right)\right|_{g \rightarrow q \bar{q}}=} & \frac{\alpha_{s}}{4 \pi^{2}} \frac{1}{x_{1}+x_{2}} f_{1}^{g}\left(x_{1}+x_{2}\right) T_{a_{1}, \bar{a}_{2}}^{l l^{\prime}}\left(\frac{x_{1}}{x_{1}+x_{2}}\right) \\
& \times \int d^{2} \boldsymbol{k} \frac{\left(\boldsymbol{k}+\frac{1}{2} \boldsymbol{r}\right)^{l}\left(\boldsymbol{k}-\frac{1}{2} \boldsymbol{r}\right)^{l^{\prime}}}{\left(\boldsymbol{k}+\frac{1}{2} \boldsymbol{r}\right)^{2}\left(\boldsymbol{k}-\frac{1}{2} \boldsymbol{r}\right)^{2}},
\end{aligned}
$$

i.e. the factors $(2 \pi)^{2}$ should be omitted on both sides.

4. Together with the surrounding text, equation (5.78) for the double parton distribution in $4-2 \epsilon$ dimensions should read:

The result for $F_{q, \bar{q}}\left(x_{i}, \boldsymbol{k}_{i}, \boldsymbol{r}\right)$ is then the same as in (5.42) with a modified kernel

$$
T_{q, \bar{q}}^{l l^{\prime}}(u ; \epsilon)=\delta^{l l^{\prime}}\left[u^{2}+(1-u)^{2}-\epsilon\right] /(1-\epsilon)
$$

times a power of $(2 \pi)^{\epsilon}$ we need not specify here.

Open Access. This article is distributed under the terms of the Creative Commons Attribution License (CC-BY 4.0), which permits any use, distribution and reproduction in any medium, provided the original author(s) and source are credited. 